\title{
The Triple Differential Cross Sections for Electron Impact Ionization of Metastable 3s State Hydrogen Atoms with Exchange Effect
}

\author{
Tania Noor ${ }^{1 *}$, Sunil Dhar ${ }^{2}$ \\ ${ }^{1}$ Department of Mathematics, Premier University, Chittagong, Bangladesh \\ ${ }^{2}$ Department of Mathematics, Chittagong University of Information and Technology, Chittagong, Bangladesh \\ Email: ^taniatima@gmail.com, sdhar03@yahoo.com
}

How to cite this paper: Noor, T. and Dhar, S. (2017) The Triple Differential Cross Sections for Electron Impact Ionization of Metastable 3s State Hydrogen Atoms with Exchange Effect. Open Journal of Microphysics, 7, 53-65.

http://dx.doi.org/10.4236/ojm.2017.73004

Received: May 1, 2017

Accepted: September 26, 2017

Published: September 30, 2017

Copyright ( 2017 by authors and Scientific Research Publishing Inc. This work is licensed under the Creative Commons Attribution International License (CC BY 4.0).

http://creativecommons.org/licenses/by/4.0/

\begin{abstract}
A final state wave function of multiple scattering theory developed by Das and seal is utilized in the present study to calculate the triple differential cross sections (TDCS) for the ionization of metastable $3 \mathrm{~S}$ state hydrogen atoms at incident electron energy of $250 \mathrm{eV}$ with the exchange effects in the asymmetric coplanar geometry for various kinematic conditions. Our present calculation results are compared with the available hydrogenic ground state experimental data and other existing theoretical results. A good qualitative agreement is shown with those of compared results of the present study specifically with hydrogenic ground state experimental data and metastable $2 \mathrm{~S}$ and $2 \mathrm{P}$ state with exchange effect results. These new results offer an extensive scope for experimental verification in such ionization process.
\end{abstract}

\section{Keywords}

Electron, Cross Sections, Ionization, Scattering

\section{Introduction}

Bethe [1] was first introduced the theoretical non-relativistic studies for the atomic ionization problems. The triple differential cross-section (TDCS) in electron hydrogen atom ionization collision has become increasingly interesting over the last four to five decades both theoretically and experimentally for relativistic [2]-[9] as well as for non-relativistic energies [10]-[21]. Triple differential cross-section (TDCS), measured in (e, 2e) coincidence experiment investigated first by Ehrhardt et al. [22] and Amaldi et al. [23]. After that many researchers have been successfully investigated such experiments in a large extent for ioniza- 
tion process theoretically both in ground state [16] [24]-[32] and metastable state [33]-[43] of atomic hydrogen by electron and positron impact.

The first theoretical calculation of direct scattering amplitude of the TDCS for the coplanar asymmetric ionization of hydrogenic metastable $2 S$-state by electrons was calculated by Vučič et al. [20] in the coplanar asymmetric geometry. The present study of triple differential cross-section (TDCS) for ionization of metastable $3 \mathrm{~S}$ state hydrogen atoms by electron exchange were never studied experimentally and theoretically. A few theoretical calculations for the TDCS of metastable $2 \mathrm{~S}$ and $2 \mathrm{P}$ [45]-[50] state hydrogen atoms by electron exchange are observed. The theoretical results of Del et al. [21], BBK model [34], and the absolute data [15] for the ionization of hydrogen atoms by electrons from ground state have been considered in the present work for comparison.

The purpose of our present work is to calculate the triple differential cross sections (TDCS) for the electron impact ionization of hydrogen atoms in the metastable $3 \mathrm{~S}$ state for coplanar asymmetric geometry with exchange effects. The present results give an interesting good qualitative fitness with the hydrogenic ground state ionization experimental data and some other hydrogenic ground state theoretical results as well as hydrogenic metastable $2 \mathrm{~S}$ state and $2 \mathrm{P}$ state results. The new observation created a new dimension in this field of research.

\section{Theory}

We have considered here the direct and exchange amplitude of the T-matrix element. The T-matrix element for ionization of hydrogen atoms by electrons [17] can be written as,

$$
T_{f i}=\left\langle\Psi_{f}^{(-)}\left(\bar{r}_{1}, \bar{r}_{2}\right)\left|V_{i}\left(\bar{r}_{1}, \bar{r}_{2}\right)\right| \Phi_{i}\left(\bar{r}_{1}, \bar{r}_{2}\right)\right\rangle
$$

Here the perturbation potential $V_{i}\left(\bar{r}_{1}, \bar{r}_{2}\right)$ is given by

$$
V_{i}\left(\bar{r}_{1}, \bar{r}_{2}\right)=\frac{1}{r_{12}}-\frac{Z}{r_{2}}
$$

For hydrogen atom nuclear charge $(Z)=1, r_{1}$ and $r_{2}$ are the distance of the two electrons from the nucleus and $r_{12}$ is the distance between the two electrons. The initial channel unperturbed wave function is,

$$
\Phi_{i}\left(\bar{r}_{1}, \bar{r}_{2}\right)=\frac{\mathrm{e}^{i \cdot \bar{p}_{2} \cdot \bar{r}_{2}}}{(2 \pi)^{3 / 2}} \varphi_{3 S}\left(\overline{r_{1}}\right)=\frac{\mathrm{e}^{i \cdot \bar{p}_{2} \cdot \bar{r}_{2}}}{(2 \pi)^{3 / 2}} \cdot \frac{1}{81 \sqrt{3 \pi}}\left(27-18 r_{1}+2 r_{1}^{2}\right) \mathrm{e}^{-\lambda_{1} r_{1}}
$$

where

$$
\varphi_{3 S}\left(\bar{r}_{1}\right)=\frac{1}{81 \sqrt{3 \pi}}\left(27-18 r_{1}+2 r_{1}^{2}\right) \mathrm{e}^{-\lambda_{1} r_{1}}
$$

and

$$
\lambda_{1}=1 / 3
$$

Equation (4) is the hydrogenic 3S-state wave function, $\bar{p}_{i}$ is the incident electron momentum, $\Psi_{f}^{(-)}\left(\bar{r}_{1}, \bar{r}_{2}\right)$ is the final three-particle scattering state wave 
function with the electrons being in the continuum with momenta $\bar{p}_{1}, \bar{p}_{2}$. Co-ordinates of the two electrons taken to be $\bar{r}_{1}$ and $\bar{r}_{2}$.

Here $\Psi_{f}^{(-)}\left(\bar{r}_{1}, \bar{r}_{2}\right)$ is approximate wave function and is given by,

$$
\begin{aligned}
& \Psi_{f}^{(-)}\left(\overline{r_{1}}, \bar{r}_{2}\right) \\
& =N\left(\bar{p}_{1}, \bar{p}_{2}\right)\left[\phi_{\bar{p}_{1}}^{(-)}\left(\bar{r}_{1}\right) \mathrm{e}^{i \cdot \bar{p}_{2} \cdot \bar{r}_{2}}+\phi_{\bar{p}_{2}}^{(-)}\left(\bar{r}_{2}\right) \mathrm{e}^{i \cdot \bar{p}_{1} \cdot \bar{r}_{1}}+\phi_{\bar{p}}^{(-)}(\bar{r}) \mathrm{e}^{i \bar{P} \cdot \bar{R}}-2 \mathrm{e}^{\overline{\bar{p}_{1}} \cdot \cdot \bar{r}_{1}+\bar{p}_{2} \cdot \bar{r}_{2}}\right] /(2 \pi)^{3}
\end{aligned}
$$

where

$$
\bar{r}=\frac{\bar{r}_{2}-\bar{r}_{1}}{2}, \bar{R}=\left(\bar{r}_{2}+\bar{r}_{1}\right) / 2, \bar{p}=\left(\bar{p}_{2}-\bar{p}_{1}\right), \bar{P}=\left(\bar{p}_{2}+\bar{p}_{1}\right)
$$

The normalization constant $N\left(\bar{p}_{1}, \bar{p}_{2}\right)$ is given by

$$
\begin{aligned}
\left|N\left(\bar{p}_{1}, \bar{p}_{2}\right)\right|^{-2}= & \mid 7-2\left[\lambda_{1}+\lambda_{2}+\lambda_{3}\right]-\left[\frac{2}{\lambda_{1}}+\frac{2}{\lambda_{2}}+\frac{2}{\lambda_{3}}\right] \\
& +\left[\frac{\lambda_{1}}{\lambda_{2}}+\frac{\lambda_{1}}{\lambda_{3}}+\frac{\lambda_{2}}{\lambda_{1}}+\frac{\lambda_{2}}{\lambda_{3}}+\frac{\lambda_{3}}{\lambda_{1}}+\frac{\lambda_{3}}{\lambda_{2}}\right] \mid
\end{aligned}
$$

here

$$
\begin{aligned}
& \lambda_{1}=\mathrm{e}^{\frac{\pi \alpha_{1}}{2}} \Gamma\left(1-i \alpha_{1}\right), \alpha_{1}=\frac{1}{P_{1}} \\
& \lambda_{2}=\mathrm{e}^{\frac{\pi \alpha_{2}}{2}} \Gamma\left(1-i \alpha_{2}\right), \alpha_{2}=\frac{1}{P_{2}} \\
& \lambda_{3}=\mathrm{e}^{\frac{\pi \alpha}{2}} \Gamma(1-i \alpha), \alpha=-\frac{1}{P}
\end{aligned}
$$

The normalization constant $N\left(\bar{p}_{1}, \bar{p}_{2}\right)$ is calculated numerically using Equation (6) and the approximated value of $N\left(\bar{p}_{1}, \bar{p}_{2}\right)$ is 1 .

$\phi_{\bar{q}}^{(-)}(\bar{r})$ is the Coulomb wave function and is given by

$$
\phi_{\bar{q}}^{(-)}(\bar{r})=\mathrm{e}^{\frac{\pi \alpha}{2}} \Gamma(1+i \alpha) \mathrm{e}^{i \bar{q} \cdot \bar{r}}{ }_{1} F_{1}(-i \alpha, 1,-i[q r+\bar{q} \cdot \bar{r}])
$$

For the electron impact ionization the parameters $\alpha_{1}, \alpha_{2}$ and $\alpha$ are given below

$$
\alpha_{1}=\frac{1}{p_{1}} \text { for } \bar{q}=\bar{p}_{1}, \quad \alpha_{2}=\frac{1}{p_{2}} \text { for } \bar{q}=\bar{p}_{2} \text { and } \alpha=\frac{1}{p} \text { for } \bar{q}=\bar{p}
$$

Equation (1) becomes,

$$
T_{f i}=N\left(\bar{p}_{1}, \bar{p}_{2}\right)\left[T_{B}+T_{B^{\prime}}+T_{i}-2 T_{P B}\right]
$$

where

$$
\begin{aligned}
& T_{B}=\left\langle\Phi_{\overline{p_{1}}}^{(-)}\left(\bar{r}_{1 ?}\right) \mathrm{e}^{\overline{\bar{p}_{2}} \cdot \overline{r_{2}}}\left|V_{i}\right| \Phi_{i}\left(\overline{r_{1}}, \bar{r}_{2}\right)\right\rangle \\
& T_{B^{\prime}}=\left\langle\Phi_{p_{2}}^{(-)}\left(\bar{r}_{2}\right) \mathrm{e}^{i \bar{p}_{1} \cdot \bar{r}_{1}}\left|V_{i}\right| \Phi_{i}\left(\bar{r}_{1}, \overline{r_{2}}\right)\right\rangle \\
& T_{i}=\left\langle\Phi_{\bar{p}}^{(-)}(\bar{r}) \mathrm{e}^{i \cdot \bar{P} \cdot \bar{R}}\left|V_{i}\right| \Phi_{i}\left(\bar{r}_{1}, \bar{r}_{2}\right)\right\rangle \\
& T_{P B}=\left\langle\mathrm{e}^{\overline{\bar{p}_{1}} \cdot \overline{\bar{r}_{1}}+\overline{p_{2}} \cdot \bar{r}_{2}}\left|V_{i}\right| \Phi_{i}\left(\bar{r}_{1}, \bar{r}_{2}\right)\right\rangle
\end{aligned}
$$


The direct scattering amplitude $f\left(\bar{p}_{1}, \bar{p}_{2}\right)$ is the determined from

$$
f\left(\bar{p}_{1}, \bar{p}_{2}\right)=-(2 \pi)^{2} T_{f i}
$$

The exchange scattering amplitude is then approximated by

$$
g\left(\bar{p}_{1}, \bar{p}_{2}\right)=f\left(\bar{p}_{2}, \bar{p}_{1}\right)
$$

After analytical calculations using Lewis Integral [44], the triple differential cross-sections (TDCS) with exchange effects is finally takes the following form,

$$
\frac{\mathrm{d}^{3} \sigma}{\mathrm{d} \Omega_{1} \mathrm{~d} \Omega_{2} \mathrm{~d} E_{1}}=\frac{p_{1} p_{2}}{p_{i}}\left[\frac{3}{4}|f-g|^{2}+\frac{1}{4}|f+g|^{2}\right]
$$

Here $E_{1}$ is the energy of the ejected electron. Hence, in our present study we have calculated the TDCS with exchange effects, given by the Equation (14) using computer programming language.

\section{Results and Discussions}

We have discussed here in this section the ionization of metastable 3S state hydrogen atoms by electrons with exchange effect. The triple differential cross-sections (TDCS) at $E_{i}=250 \mathrm{eV}$ incident energy with the ejected electron energy of $5 \mathrm{eV}$ is calculated. The results of exchange effects are displayed by the Figure $1\left(\theta_{2}=3^{\circ}\right)$, Figure $2\left(\theta_{2}=15^{\circ}\right)$, Figure $3\left(\theta_{2}=25^{\circ}\right)$, Figure $4\left(\theta_{2}=5^{\circ}\right)$, Figure $5\left(\theta_{2}=7^{\circ}\right)$, Figure $6\left(\theta_{2}=9^{\circ}\right)$, Figure $7\left(\theta_{2}=11^{\circ}\right)$, Figure $8\left(\theta_{2}=15^{\circ}\right)$ and Figure $9\left(\theta_{2}=20^{\circ}\right)$. We consider here the incident electron energy is $E_{i}=250 \mathrm{eV}$ for some varied ejected angles $\left(\theta_{1}\right)$ and for a fixed scattering angle $\left(\theta_{2}\right)$. The ejection angle $\theta_{1}$ varies from $0^{\circ}$ to $360^{\circ}$. We consider here the recoil regions of the following figures from $\theta_{1}\left(0^{\circ}-150^{\circ}\right)$ and $\phi=0^{\circ}$ on other hand the binary region is from $\theta_{1}\left(150^{\circ}-360^{\circ}\right)$ and $\phi=180^{\circ}$.

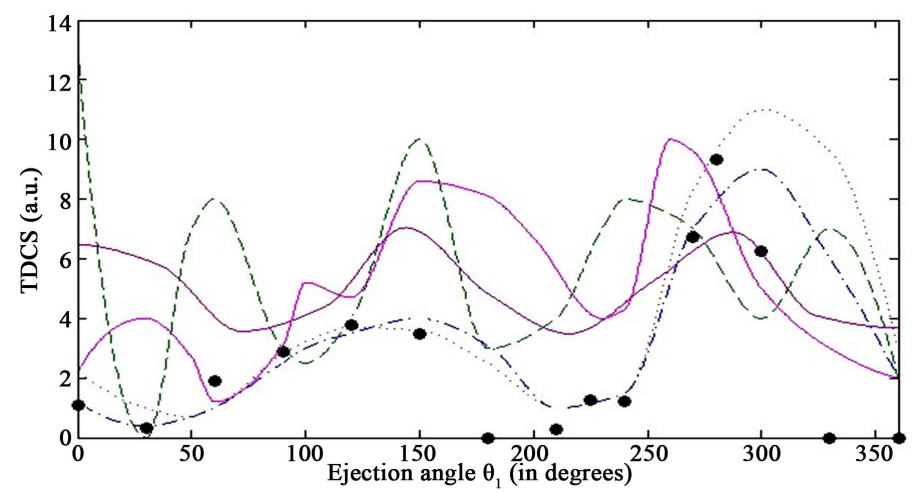

Figure 1. Triple differential cross sections (TDCS) for ionization of atomic hydrogen by $250 \mathrm{eV}$ electron impact for $\theta_{2}=3^{\circ}$ vary against the ejected electron $\theta_{1}$ relative to the incident electron direction. The ejected electron energy is $E_{1}=5 \mathrm{eV}$. Theory: Full filled double curve reflects the present calculation of $3 \mathrm{~S}$ state with exchange effect. Full single curve represent the first born $3 \mathrm{~S}$ state result [48]. Dash curve shows the second born $2 \mathrm{P}$ state result [47]. Short dash curve focuses hydrogenic ground state second born result [21]; Dash-dotted curve shows hydrogenic ground state BBK model [34] and filled round shows hydrogenic ground state experiments [15]. 


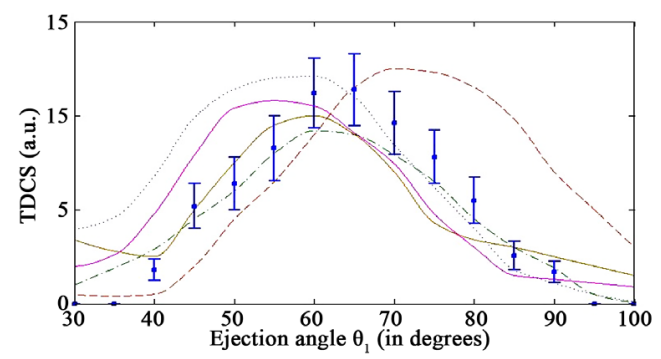

Figure 2. Triple differential cross sections (TDCS) for ionization of atomic hydrogen by $250 \mathrm{eV}$ electron impact for $\theta_{2}=15^{\circ}$ vary against the ejected electron $\theta_{1}$ relative to the incident electron direction. The ejected electron energy is $E_{1}=50 \mathrm{eV}$. Theory: Full curve (m) reflects the present calculation of $3 \mathrm{~S}$ state with exchange effect. Full single curve (red) represent the first born $3 \mathrm{~S}$ state result [48]. Dash curve shows the second born $2 \mathrm{P}$ state result [47]. Short dash curve focuses hydrogenic ground state second born result [21]; Dash-dotted curve shows hydrogenic ground state BBK model [34], dash-dotted curve reflects the second born $2 S$-state result [38] and square shows hydrogenic ground state experiments [15].

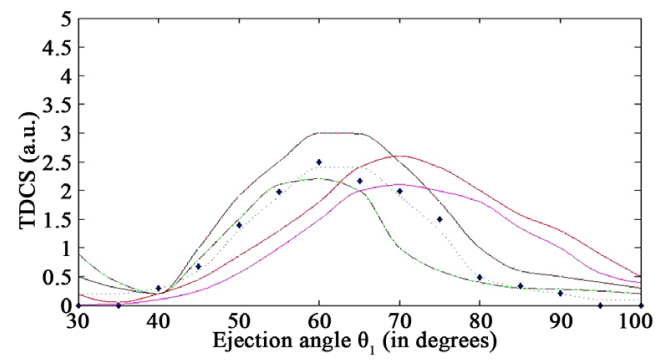

Figure 3. Triple differential cross sections (TDCS) for ionization of atomic hydrogen by $250 \mathrm{eV}$ electron impact for $\theta_{2}=25^{\circ}$ vary against the ejected electron $\theta_{1}$ relative to the incident electron direction. The ejected electron energy is $E_{1}=50 \mathrm{eV}$. Theory: Full curve (m) reflects the present calculation of $3 \mathrm{~S}$ state with exchange effect. Full single curve (red) represent the first born $3 \mathrm{~S}$ state result [48]. Dash curve shows the second born $2 \mathrm{P}$ state result [47]. Short dash curve focuses hydrogenic ground state second born result [21]; Dash-dotted curve shows hydrogenic ground state BBK model [34], dash-dotted curve reflects the second born 2S-state result [38] and square shows hydrogenic ground state experiments [15].

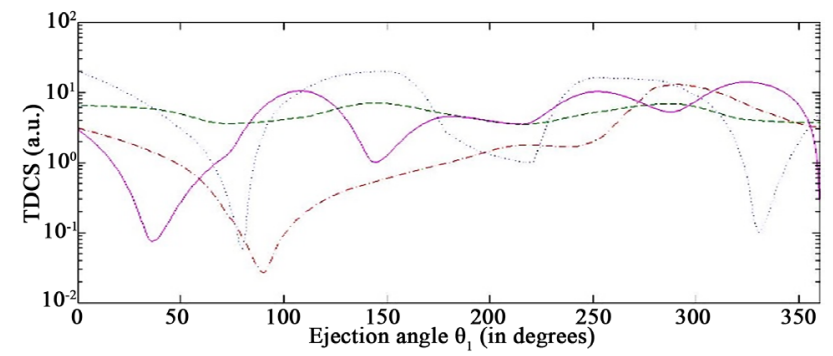

Figure 4. Triple differential cross sections (TDCS) for ionization of atomic hydrogen by $250 \mathrm{eV}$ electron impact with exchange effects for $\theta_{2}=5^{\circ}$ vary against the ejected electron $\theta_{1}$ relative to the incident electron direction. The ejected electron energy is $E_{1}=5 \mathrm{eV}$. Theory: Full curve reflects the present calculation. Dash curve represent the first born 3Sstate result [48]. Short dash curve shows the second born 2P-state result [47]. Dash-dot curve exhibits the hydrogenic $2 \mathrm{~S}$-state result [38]. 


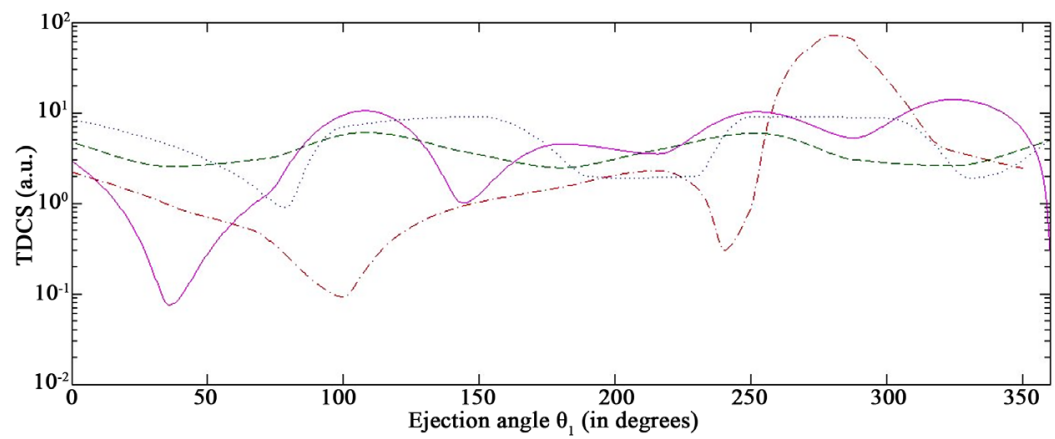

Figure 5. Triple differential cross sections (TDCS) for ionization of atomic hydrogen by $250 \mathrm{eV}$ electron impact with exchange effects for $\theta_{2}=7^{\circ}$ vary against the ejected electron $\theta_{1}$ relative to the incident electron direction. The ejected electron energy is $E_{1}=5 \mathrm{eV}$. Theory: Full curve reflects the present calculation. Dash curve represent the first born 3Sstate result [48]. Short dash curve shows the second born 2P-state result [47]. Dash-dot curve exhibits the hydrogenic 2 S-state result [38].

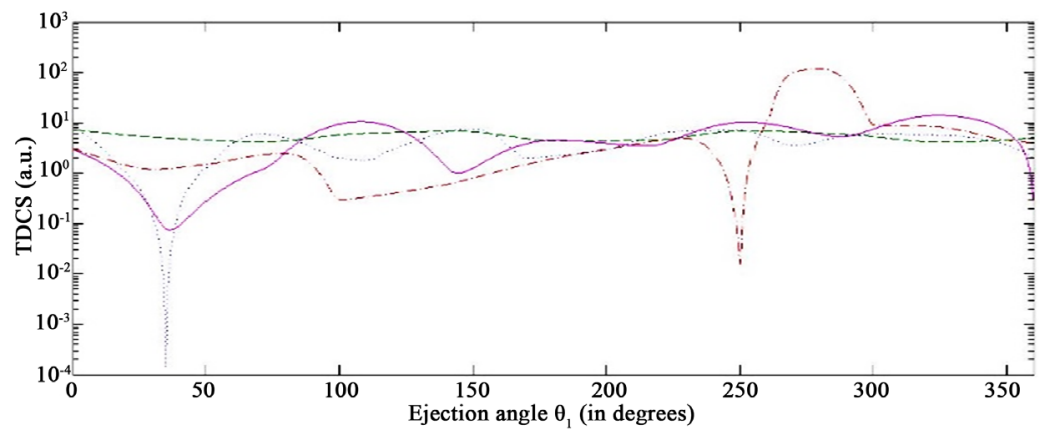

Figure 6. Triple differential cross sections (TDCS) for ionization of atomic hydrogen by $250 \mathrm{eV}$ electron impact with exchange effects for $\theta_{2}=9^{\circ}$ vary against the ejected electron $\theta_{1}$ relative to the incident electron direction. The ejected electron energy is $E_{1}=5 \mathrm{eV}$. Theory: Full curve reflects the present calculation. Dash curve represent the first born 3S-state result [48]. Short dash curve shows the second born 2P-state result [47]. Dash-dot curve exhibits the hydrogenic 2S-state result [38].

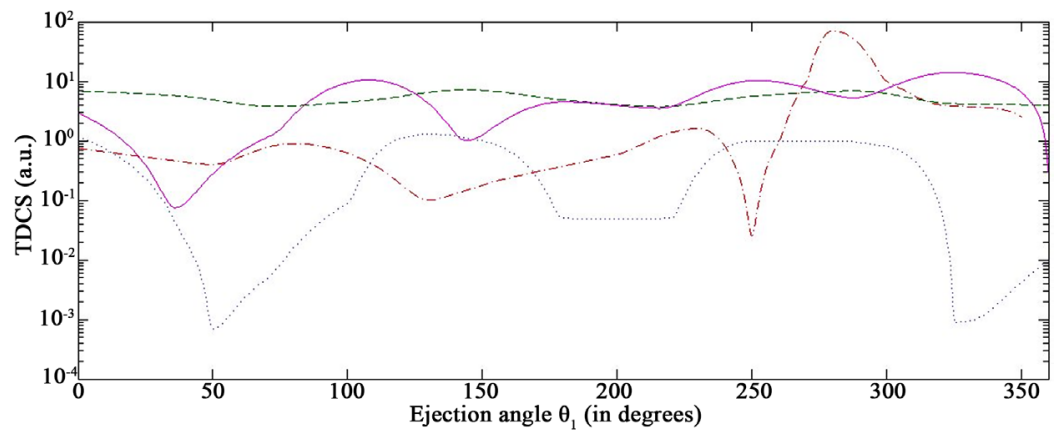

Figure 7. Triple differential cross sections (TDCS) for ionization of atomic hydrogen by $250 \mathrm{eV}$ electron impact with exchange effects for $\theta_{2}=11^{\circ}$ vary against the ejected electron $\theta_{1}$ relative to the incident electron direction. The ejected electron energy is $E_{1}=5 \mathrm{eV}$. Theory: Full curve reflects the present calculation. Dash curve represent the first born 3S-state result [48]. Short dash curve shows the second born 2P-state result [47]. Dash-dot curve exhibits the hydrogenic 2S-state result [38]. 


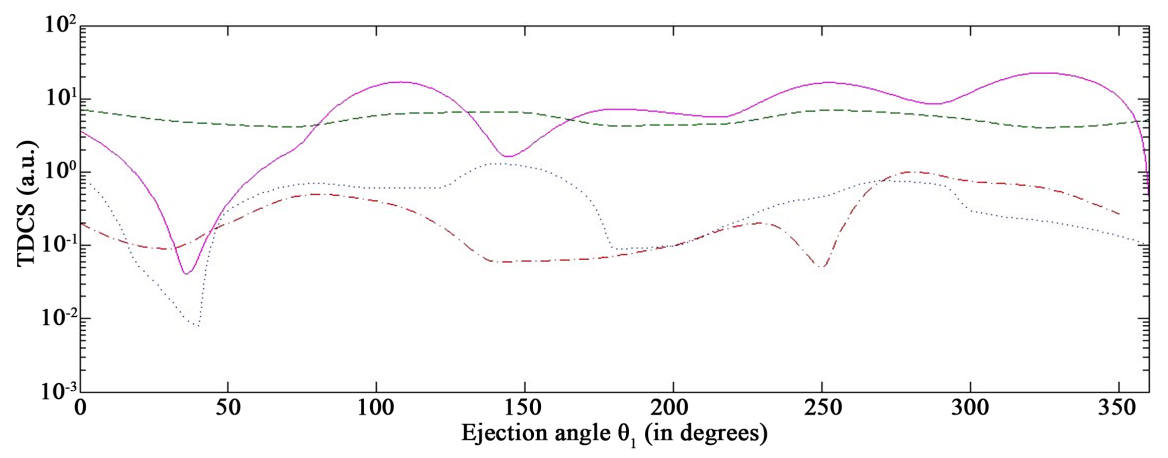

Figure 8. Triple differential cross sections (TDCS) for ionization of atomic hydrogen by $250 \mathrm{eV}$ electron impact with exchange effects for $\theta_{2}=15^{\circ}$ vary against the ejected electron $\theta_{1}$ relative to the incident electron direction. The ejected electron energy is $E_{1}=5 \mathrm{eV}$. Theory: Full curve reflects the present calculation. Dash curve represent the first born 3Sstate result [48]. Short dash curve shows the second born 2P-state result [47]. Dash-dot curve exhibits the hydrogenic $2 \mathrm{~S}$-state result [38].

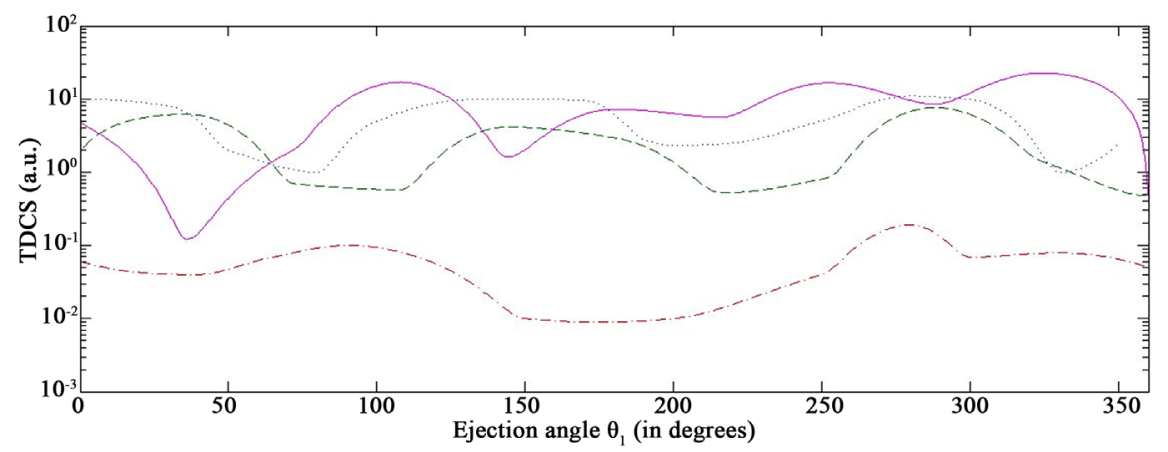

Figure 9. Triple differential cross sections (TDCS) for ionization of atomic hydrogen by $250 \mathrm{eV}$ electron impact with exchange effects for $\theta_{2}=20^{\circ}$ vary against the ejected electron $\theta_{1}$ relative to the incident electron direction. The ejected electron energy is $E_{1}=5 \mathrm{eV}$. Theory: Full curve reflects the present calculation. Dash curve represent the first born 3Sstate result [48]. Short dash curve shows the second born 2P-state result [47]. Dash-dot curve exhibits the hydrogenic 2 S-state result [38].

Electron-hydrogen ionization from ground state theoretical results of Dal et al. [21], the BBK model of Brauner et al. [34] and the experimental results of Ehrhardt et al. [15] are presented here for comparisons. The earlier works of hydrogenic 2S-state [38] ionization results. The recent works on hydrogenic 2P-state [47] ionization results are also exhibited here for comparison with our present work. We have considered here the triple differential cross-sections (TDCS) with exchange effects for the ionization of metastable 3S-state hydrogen atoms by electrons for the incident electron energy of $E_{i}=250 \mathrm{eV}$ and ejected electron energies $E_{1}=5 \mathrm{eV}$ and $50 \mathrm{eV}$. The final continuum state of the present work shows a similar but shifted amplitude in the recoil lobe position as the earlier result of hydrogenic ground state second born approximation [21].

In Table 1 we figure a comparison data for our present result of triple differential cross sections for ionization of hydrogenic $3 \mathrm{~S}$ state by electron impact with exchange effects with the $2 \mathrm{P}$ state exchange results. 
Table 1. Triple differential cross sections (TDCS) for ionization of atomic hydrogen atoms by electron impact with exchange effects are obtained by Equation (14) at metastable 3S-state. The incident energy is $250 \mathrm{eV}$, the scattering angle is $\theta_{2}=9 \mathrm{n}$ and the ejected electron energy is $E_{1}=5 \mathrm{eV}$. In Table $1 \mathrm{~B} 1(2 \mathrm{P})$ : is the compared $2 \mathrm{P}$ state exchange results and $\mathrm{B} 2(3 \mathrm{~S})$ : is the present results.

\begin{tabular}{ccc}
\hline Ejected angles $\left(\theta_{1}\right)$ & B1 (2P) & B2(3S) \\
\hline 0 & 5.6923 & 1.1151 \\
36 & 3.625 & 5.0858 \\
72 & 1.46154 & 0.0752 \\
108 & 6.4231 & 5.0380 \\
144 & 6.8077 & 5.0135 \\
180 & 8.3077 & 7.2945 \\
216 & 6.6077 & 6.1394 \\
252 & 7.19231 & 5.1394 \\
288 & 7.7769 & 10.0288 \\
324 & 5.15 & 5.0395 \\
360 & 5.38462 & 0.0250 \\
\hline
\end{tabular}

In Figure 1 we consider the ejected electron energy $E_{1}=5 \mathrm{eV}$ with a fixed scattering angle $\theta_{2}=3^{\circ}$ and the incident electron energy is $250 \mathrm{eV}$. It is exciting to observe that our present results show a good qualitative agreement with the compared results of present first born result [48], the hydrogenic ground state result of BBK model [34], the second born approximation [21], the experimental data [15] and the second born experiment of 2P-state [47] exchange effects results. Our present result shows a fall in the recoil region and two prominent peaks in binary region.

We consider for Figure 2 the ejected electron energy $E_{i}=250 \mathrm{eV}$, scattered electron energy $E_{1}=50 \mathrm{eV}$ and scattered angle $\theta_{2}=15^{\circ}$. We also consider the ejected electron angle $\theta_{1}$ from $30^{\circ}$ to $100^{\circ}$. In this figure our present results gives small magnitude compared with the previous theoretical results [21] [38] [47] [48] and shows a good qualitative similarity with the experimental results [15] [34].

In a similar way Figure 3 shows us the $3 \mathrm{~S}$ metastable state with exchange effects for ejected electron energy $E_{i}=250 \mathrm{eV}$, scattered electron energy $E_{1}=50 \mathrm{eV}$ and scattered angle $\theta_{2}=25^{\circ}$. As we increase our scattering angle $\left(\theta_{2}\right)$ the peak of our present study shows a smaller magnitude with hydrogenic ground state second born results [21].

From Figures 4-9 we compared the present exchange effects results with previous theoretical results like $2 \mathrm{P}$ and $2 \mathrm{~S}$ metastable states [38] [47]. We also include here the present first Born result [48]. The scattering angle varies from $\theta_{2}=5^{\circ}$ (Figure 4), $\theta_{2}=7^{\circ}$ (Figure 5), $\theta_{2}=9^{\circ}$ (Figure 6), $\theta_{2}=11^{\circ}$ (Figure 7), $\theta_{2}=15^{\circ}$ (Figure 8), $\theta_{2}=20^{\circ}$ (Figure 9) in the fixed incident energy 
$E_{i}=250 \mathrm{eV}$, ejected energy $E_{1}=5 \mathrm{eV}$ and the ejected electron angle is vary from $\left(\theta_{1}=0^{\circ}\right.$ to $\left.360^{\circ}\right)$.

Figure 4 and Figure 5 our present result shows a good agreement of our present result with $2 \mathrm{~S}$-state metastable exchange results [38]. The figure displayed a deep lobed peak structure which shows a good qualitative similarity with the compared results [38] [47] [48].

When we increase our scattering angle in Figure 6 our present result and the present first Born result remains same and give a good qualitative agreement with the compared 2P state exchange result [47].

In Figure 7 our present TDCS exchange curve shows a very interesting result. It exhibits two falls in recoil region whereas the compared $2 S$ state exchange result [38] there is only one fall in the recoil region. The result shows a bit different from 2P state exchange result [47].

In the Figure 8 and Figure 9 the magnitude of the present results smaller than the present first Born results [48]. The characteristic features of the cross section curves of the present calculation shows a good improvement comparing with the previous results as the scattering angles are increasing.

The present result of 3S-state exchange effects gives a good qualitative improvement comparing with the previous $2 \mathrm{~S}$ and $2 \mathrm{P}$ state exchange effect results. The present result also show a similar conduct with the hydrogenic ground state result [21] in the binary region. Moreover there needs more experimental works in this field for further investigation.

Finally, the scattering mechanism for the ionization of metastable $3 \mathrm{~S}$ state with exchange effects for $250 \mathrm{eV}$ incident electron energy is presented here in this study. The scattered electrons are described by a plane wave in the first Born term of Equation (5) whereas the ejected electrons are defined by a Coulomb wave. In the second term of the Equation (5) the scattered electrons are defined by the Coulomb wave while the ejected electrons are defined by the plane wave. The projectile electron interaction appeared in the third term shows almost similar behavior in the final channel wave function. The fourth term represents two plane waves for both ejected and scattered particles. The above results gives us a strong view of peaks both in recoil region and binary region. We can conclude that the present peak values gives us a good agreement with our compared experimental results as well as the theoretical results. In Our present study the measurements of peak values gives us the encouragement for further research in this field of interest. Moreover it needs more experimental works in this field for further investigation.

\section{Conclusion}

Our present calculation on the triple differential cross sections for ionization of atomic hydrogen by electron impact with exchange effects in metastable 3S-state exposes a thinkable additional structure of the cross-section curves for small momentum transfer in the ionization of the hydrogen atoms. The final state 
wave function $\psi_{f}^{(-)}\left(\bar{r}_{1}, \bar{r}_{2}\right)$ of Das and Seal gives a good qualitative result with the hydrogenic ground state experiment as well as with the BBK model of ground state hydrogen atoms. For good qualitative agreement, the present study are very encouraging for the future experiments which may play a vital role to give interesting and significant results in this field of research.

\section{Acknowledgements}

The computational works have been performed in the Simulation Lab of the Department of Mathematics, Chittagong University of Engineering and Technology Chittagong-4349, Bangladesh.

\section{References}

[1] Bethe, H. (1930) A Zur Theorie des Durchgangs schneller Korpuskularstrahlen durch Materie. Asymmetric Ann Phys, 5, 325. Handbuch der Physik Ed. A Smekal Aufbau Der Zusammenhangenden Materie. 1933, 24:273.

[2] Madison, D.H. and Merzbacher, E. (1975) Semi Classical Transition Probabilities for the Electron-Impact Excitation of Hydrogeic Ions in Dense Plasma. Academic Press, New York, 1.

[3] Das, J.N. (1972) Advances in Atomic and Molecular Physics. 1 Nuovo Cimento B, $12,17$.

[4] Das, J.N. and Chakraborty, S. (1985) An Improved Calculations for The Inner-Shell Ionization Problems. Physics Letters A, 32, 176.

[5] Jakubasa-Amundsen, D.H. Relativistic Theory for K-Shell Ionization by Fast Electrons. Z. Phys., 1989, D11, 305; Journal of Physics, 1992, B25, 1297.

[6] Keller, S., Dreizler, R.M., Ancarni, L.U., Watters, H.R.J., Ast, H. and Whelan, C.T. (1996) Theoretical Analysis of the Relativistic First Order Born Approximation for Inner Shell (e,2e) Processes. Z. Phys., D37, 191.

[7] Schüle, E and Nakel, W. (1982) Triply-Differential Cross Section for K-Shell Ionization of Silver by Relativistic Electron Impact. Journal of Physics, B15, L639.

[8] Ruoff, H. and Nakel, W. (1987) Absolute Triply Differential Cross Section for K-Shell Ionization by Relativistic Electron Impact for High Atomic Number. Journal of Physics, B20, 2299.

[9] Bonfert, J., Graf, H. and Nakel, W. (1991) Relativistic (e,2e) Collisions on Atomic Inner Shells in Symmetric Geometry. Journal of Physics, B24, 1423.

[10] Massey H.S.W. and Mohr, C.B.O. (1933) Gaseous Reactions Involving Positronium. Proceedings of the Physical Society. Section A, 67, 695. https://doi.org/10.1088/0370-1298/67/8/306

[11] Ehrhardt, H., Hesselbacher, K.H., Jung, K. and Willman, K. (1972) Collisional Ionization of Helium by Slow Electrons. Journal of Physics B, 5, 1559.

[12] Lahmann, B., McCarthy, I.E., Stelbovics, A.T. and Weigold, E. (1984) Electron-Impact Ionization of Atomic Hydrogen: Comparison of Asymmetric (e, 2e) Measurements with Theories. Physical Review A, 30, 758. https://doi.org/10.1103/PhysRevA.30.758

[13] Weigold, E., Noble, C.J., Hood, S.T. and Fuss, I. (1979) Electron Impact Ionization of Atomic Hydrogen: Experimental and Theoretical (e, 2e) Differential Cross Section. Journal of Physics B: Atomic, Molecular and Optical Physics, 12, 291. 
https://doi.org/10.1088/0022-3700/12/2/019

[14] Joachain, C.J. and Piraux, B. (1986) Theory of Coplanar Asymmetric (e, 2e) Reactions in Helium. Comments on Atomic and Molecular Physics, 17, 261.

[15] Ehrhardt, H., Knoth, G., Schlemmer, P. and Jung, K. (1986) Differential Cross Sections of Direct Single Electron Impact Ionization. Zeitschrift für Physik D, 1, 3. https://doi.org/10.1007/BF01384654

[16] Byron, W.F., Joachain, J.C. and Piraux, B. (1980) Triple Differential Cross Sections for the Ionization of Atomic Hydrogen by Fast Electrons: A Second Born Treatment. Journal of Physics B: Atomic, Molecular and Optical Physics, 13, L673.

[17] Das, J.N. and Seal, S. (1993) Electron-Hydrogen-Atom Ionization Collision at Intermediate (21_0-201_0) and High ( $\left.\geq 201 \_0\right)$ Energies. Physical Review A, 47, 2978. https://doi.org/10.1103/PhysRevA.47.2978

[18] Lahmann-Bennani, A. (1991) Recent Developments and Ne Trends in (e, 2e) and (e, 3e) Studies. Journal of Physics B, 24, 2401. https://doi.org/10.1088/0953-4075/24/10/001

[19] Das, J.N. and Dhar, S. (1999) Energy Spectrum of Ejected Electrons in Ionization of Hydrogen Atoms by Electrons. Pramana Journal of Physics, 47, 263-269.

[20] Vucic, S., Potvliege, R.M. and Joachain, C.J. (1987) Second Born Triple-Differential Cross Sections for the Coplanar Asymmetric Ionization of $\mathrm{H}(2 \mathrm{~S})$ by Fast Electrons. Physical Review A, 35, 1446. https://doi.org/10.1103/PhysRevA.35.1446

[21] Dal Cappello, C., Haddadou, A., Menas, F. and Roy, A.C. (2011) The Second Born Approximation fr the Single and Double Ionization of Atoms by Electrons and Positrons. Journal of Physics B: Atomic, Molecular and Optical Physics, 44, Article ID: 015204. https://doi.org/10.1088/0953-4075/44/1/015204

[22] Ehrhardt, H., Schulz, M., Tekkat, T. and Willmann, K. (1969) Differential Cross Sections of Direct Single Electron Impact Ionization. Physical Review Letters, $22,89$.

[23] Amaldi, U., Egidi, A., Marconero, R. and Pizzela, G. (1969) Use of a to Channeltron Coincidence in a New Line of Research in Atomic Physics. Review of Scientific Instruments, 40, 1001. https://doi.org/10.1063/1.1684135

[24] Byron, F.W., Joachain, C.J. and Piraux, B. (1886) Theory of Coplanar Asymmetric (e, 2e) Reactions in Helium. Journal of Physics B: Atomic, Molecular and Optical Physics, 19, 1201. https://doi.org/10.1088/0022-3700/19/8/015

[25] Byron, F.W., Joachain, C.J. and Piraux, B. (1985) Triple Differential Cross Sections for the Ionization of Atomic Hydrogen by Fast Electrons: A Second Born Treatment. Journal of Physics B: Atomic, Molecular and Optical Physics, 18, 3203.

[26] Das, J.N. (1990) Momentum-Space Analysis of Scattering States with Possible Application to Atomic Ionization. Physical Review A, 42, 1376. https://doi.org/10.1103/PhysRevA.42.1376

[27] Das, J.N. and Seal, S. (1993) Electron-Hydrogen-Atom Ionization Collision at Intermediate (21_0-201_0) and High ( $\left.\geq 201 \_0\right)$ Energies. Physical Review A, 40, 253.

[28] Das, J.N. and Dhar, S. (1999) Energy Spectrum of Ejected Electrons in Ionization of Hydrogen Atoms by Electrons. Pramana Journal of Physics, 53, 869. https://doi.org/10.1007/s12043-999-0121-9

[29] Jones, S. and Madison, D.H. (2000) Ionization of Hydrogen Atoms by Fast Electrons. Physical Review A, 62, Article ID: 042701.

https://doi.org/10.1103/PhysRevA.62.042701 
[30] Jones, S. and Madison, D.H. (2002) Scaling Behavior of the Fully Differential Cross Section for Ionization of Hydrogen Atoms by the Impact of Fast Elementary Charged Particles. Physical Review A, 65, Article ID: 052727. https://doi.org/10.1103/PhysRevA.65.052727

[31] Li, S., Berakdar, J., Zhang, S.T. and Chen, J. (2005) Laser Assisted (e, 2e) Reaction in One Electron Atoms and Ions. Journal of Physics B: Atomic, Molecular and Optical Physics, 38, 1291. https://doi.org/10.1088/0953-4075/38/8/017

[32] Das, J.N. and Dhar, S. (1998) Energy Spectrum of Scattered Electrons in K-Shell Ionization of Medium to Heavy Atoms by Relativistic Electrons. Journal of Physics B: Atomic, Molecular and Optical Physics, 31, 2355. https://doi.org/10.1088/0953-4075/31/10/021

[33] Bransden, B.H. and Joachain, C.J. (1983) Physics of Atoms and Molecules. 2nd Edition, Pearson Education, New York.

[34] Brauner, M., Briggs, J.S. and Klar, H. (1989) Triply-Differential Cross Sections for Ionization of Hydrogen Atoms by Electrons and Positrons. Journal of Physics B: Atomic, Molecular and Optical Physics, 22, 2265-2287.

https://doi.org/10.1088/0953-4075/22/14/010

[35] Ray, H. and Roy, A.C. (1988) Triply Differential Cross Sections for the Coplanar Asymmetric Ionization of $\mathrm{H}(2 \mathrm{~S})$ by Fast Electrons. Journal of Physics B: Atomic, Molecular and Optical Physics, 21, 3243. https://doi.org/10.1088/0953-4075/21/19/014

[36] Hafid, H., Joulakian, B. and Cappelo, C.D. (1993) Theoretical Study of the Differential Cross Section of the Ionization of Hydrogen (2S) by Electron Impact. Journal of Physics B: Atomic, Molecular and Optical Physics, 26, 3415. https://doi.org/10.1088/0953-4075/26/19/028

[37] Das, J.N and Seal, S. (1993) Electron-Hydrogen-Atom Ionization Collision at Intermediate (21_0-201_0) and High ( $\left.\geq 201 \_0\right)$ Energies. Physical Review A, 47, 2978. https://doi.org/10.1103/PhysRevA.47.2978

[38] Dhar, S. (1996) Electron Impact Ionization of Metastable 2S-State Hydrogen Atoms. Australian Journal of Physics, 49, 937. https://doi.org/10.1071/PH960937

[39] Das, J.N. and Dhar, S. (1998) Calculation of Triple Differential Cross-Sections of K-Shell Ionization of Medium-Heavy Atoms by Electrons for Symmetric Geometry. Pramana Journal of Physics, 51, 751. https://doi.org/10.1007/BF02832607

[40] Kyle, H.L. and Omidvar, K. (1968) Passage of Charged Particles through Matter. Physical Review, 176, 164. https://doi.org/10.1103/PhysRev.176.164

[41] Berakdar, J., Engelns, A. and Klar, H. (1996) Oriented and Aligned Two-Electron Continue. Journal of Physics B: Atomic, Molecular and Optical Physics, 29, 1109. https://doi.org/10.1088/0953-4075/29/5/019

[42] Karnali, M.Z.M., Ratnavelu, K. and Zhou, Y. (2008) Electron Impact Excitation of $2 \mathrm{P}$ and $3 \mathrm{P}$ States of Hydrogen at Intermediate Energies. The European Physical Journal D, 46, 267-279. https://doi.org/10.1140/epjd/e2007-00317-0

[43] Qi, Y.Y., Ning, L.N., Wang, J.G. and Qu, Y.Z. (2013) Plasma Effect on Fast-Electron-Impact-Ionization from 2P State of Hydrogen-Like Ions. Physics of Plasmas, 20, Article ID: 123301. https://doi.org/10.1063/1.4833616

[44] Lewis, R.R. (1956) Potential Scattering of High-Energy Electrons in Second Born Approximation. Physical Review, 102, 537. https://doi.org/10.1103/PhysRev.102.537

[45] Dhar, S. and Nahar, N. (2015) Electron Impact Ionization of Metastable 2P-State Hydrogen Atoms in the Coplanar Geometry. Results in Physics, 5, 3-8. 
[46] Dhar, S. and Nahar, N. (2015) Energy Spectrum of Ejected Electrons of H (2P) Ionization by Electrons in Coplanar Asymmetric Geometry. American Journal of Modern Physics, 4, 132. https://doi.org/10.11648/j.ajmp.20150403.15

[47] Dhar, S. and Nahar, N. (2016) Triple Differential Cross-Sections for the Ionization of Metastable 2P-State Hydrogen Atoms by Electrons with Exchange Effects. Pramana Journal of Physics, 87, 69.

[48] Dhar, S., Noor, T. and Chowdhury, F.S. (2015) Electron Impact Ionization of Metastable 3S-State Hydrogen Atoms by Electrons in Coplanar Geometry. American Journal of Modern Physics, 4, 361-366. https://doi.org/10.11648/j.ajmp.20150406.11

[49] Dhar, S., Akter, S. and Nahar, N. (2016) The First Born Triple Differential Cross Sections for Ionization of $\mathrm{H}(3 \mathrm{P})$ by Electron Impact in the Asymmetric Coplanar Geometry. Open Journal of Medical Psychology, 6, 15-23.

https://doi.org/10.4236/ojm.2016.61002

[50] Bartschat, K. (2002) Electron-Impact Excitation of Helium from the $1 \mathrm{~S}$ and $2 \mathrm{~S}$ States. Journal of Physics B: Atomic, Molecular and Optical Physics, 35, L527.

https://doi.org/10.1088/0953-4075/35/23/104

\section{Submit or recommend next manuscript to SCIRP and we will provide best service for you:}

Accepting pre-submission inquiries through Email, Facebook, LinkedIn, Twitter, etc. A wide selection of journals (inclusive of 9 subjects, more than 200 journals)

Providing 24-hour high-quality service

User-friendly online submission system

Fair and swift peer-review system

Efficient typesetting and proofreading procedure

Display of the result of downloads and visits, as well as the number of cited articles

Maximum dissemination of your research work

Submit your manuscript at: http://papersubmission.scirp.org/

Or contact ojm@scirp.org 